\title{
Perioperative management of patients undergoing thoracic endovascular repair
}

\author{
Subhasis Chatterjee ${ }^{1,2,3}$, Ourania Preventza ${ }^{2,3,4}$, Vicente Orozco-Sevilla $^{2,3,4}$, Joseph S. Coselli $^{2,3,4}$ \\ ${ }^{1}$ Division of General Surgery, Michael E. DeBakey Department of Surgery, Baylor College of Medicine, Houston, Texas, USA; ${ }^{2}$ Division of \\ Cardiothoracic Surgery, Michael E. DeBakey Department of Surgery, Baylor College of Medicine, Houston, Texas, USA; ${ }^{3}$ Department of \\ Cardiovascular Surgery, Texas Heart Institute, Houston, Texas, USA; ${ }^{4}$ Department of Cardiovascular Surgery, CHI St Luke's Health-Baylor St \\ Luke's Medical Center, Houston, Texas, USA \\ Correspondence to: Subhasis Chatterjee, MD. One Baylor Plaza, BCM390, Houston, TX 77030, USA. Email: Subhasis.Chatterjee@bcm.edu.
}

\begin{abstract}
Thoracic endovascular aortic repair (TEVAR) is a less invasive method for treating thoracic and some thoracoabdominal aortic aneurysms, dissections of the thoracic aorta and blunt traumatic aortic injury, compared with conventional open surgery. Maximizing the likelihood of a successful outcome requires diligent multidisciplinary (surgical, critical care, nursing, pharmacy, nutrition and physical therapy) perioperative care. In this article, we discuss fundamentals for managing patients after endovascular aortic aneurysm repair. These principles focus on the transition between the operating room and the intensive care unit, prevention and management of spinal cord deficits (SCD), and vital neurological, respiratory, cardiovascular, renal, gastrointestinal and hematological concerns. The better the care team understands the expected postoperative course, the earlier that deviations can be recognized and the more likely that successful rescue can be achieved to reduce the incidence and severity of adverse outcomes. Achieving optimal results after TEVAR requires attention to detail across the preoperative, intraoperative and postoperative phases of care.
\end{abstract}

Keywords: Thoracic aortic endograft; thoracic aortic aneurysm; perioperative care; cerebrospinal fluid drainage

Submitted Apr 26, 2021. Accepted for publication Sep 25, 2021.

doi: 10.21037/acs-2021-taes-74

View this article at: https://dx.doi.org/10.21037/acs-2021-taes-74

\section{Introduction}

Thoracic endovascular aortic repair (TEVAR) is less physiologically demanding on patients than conventional open surgical repair. Nonetheless, significant morbidity and mortality can result from the procedure itself and patient comorbidities. Here, we discuss key perioperative management principles for the care of TEVAR patients, focusing on immediate postoperative management.

TEVAR can be used to treat fusiform and saccular descending thoracic aortic aneurysms (DTAAs), acute and chronic type B aortic dissection (TBAD) and blunt thoracic aortic injury. In more advanced forms, TEVAR with custom-made branched or fenestrated endografts can treat extensive thoracoabdominal aortic aneurysms (TAAAs). Each of these conditions reflects a different level of acuity and complexity. The better the care team understands the expected post-TEVAR course, the earlier that deviations can be recognized and the more likely that successful rescue from adverse events can be achieved (1).

\section{Preoperative considerations}

Individualized preoperative cardiovascular risk assessment is performed in elective settings consistent with current guidelines (2) focusing on age and physiological reserve, risk stratification, antiplatelet and anticoagulant medication cessation, tobacco cessation if necessary and optimization of major neurological, cardiovascular, respiratory and renal comorbidities, to reduce modifiable risks. Major preoperative comorbidities should be evaluated according to 
acuity of presentation and clinical indication for TEVAR (3).

Carotid duplex ultrasonography evaluates for concomitant carotid artery disease in patients who are $>60$ years old or have a $\geq 20$ pack-year smoking history. Carotid anatomy is especially relevant if left subclavian artery (LSA) coverage is necessary. Symptomatic carotid disease is treated before elective TEVAR. The size and takeoff of the vertebral arteries and the presence of a patent left internal mammary artery after previous coronary artery bypass grafting should be taken into account during revascularization before LSA coverage.

Patients with borderline pulmonary function should be optimized with pulmonary rehabilitation preoperatively before elective TEVAR. This includes exercise, weight loss, incentive spirometry training, bronchodilator therapy and mandatory tobacco cessation for at least 2-3 weeks. Any existing pleural effusion can be drained during the TEVAR.

Patients with acute TBAD needing urgent TEVAR for malperfusion are managed with anti-impulse therapy to maintain a systolic blood pressure $<120 \mathrm{mmHg}$ and a heart rate $<70$ beats per minute. Intravenous beta blockers (e.g., esmolol, labetalol) and vasodilators (e.g., nicardipine) are used while diagnostic and laboratory tests are performed. Patients with reduced creatinine clearance require adequate hydration before surgery or computed tomography (CT) imaging to reduce the risk of acute kidney injury (AKI).

Antiplatelet agents (i.e., $\mathrm{P} 2 \mathrm{Y}_{12}$ inhibitors, aspirin) and antithrombotic therapy (i.e., warfarin, non-vitamin $\mathrm{K}$ oral anticoagulants) are discontinued without bridging therapy one week before surgery to allow for cerebrospinal fluid (CSF) drainage (4). This practice is individualized in patients with recent coronary stents or those unlikely to need CSF drainage. Patients requiring an urgent operation while receiving warfarin therapy are administered intravenous vitamin $\mathrm{K}$ or prothrombin complex concentrate to reverse anticoagulation, or else a CSF drain is not placed preoperatively.

After the procedure is completed during the recovery period, clear communication between the surgical, anesthesia, critical care and nursing teams are vital (5). Communication should focus on the extent of stent graft coverage, hemodynamic disturbances, airway or pulmonary concerns, whether planned or unplanned concomitant procedures were performed, anatomical concerns for malperfusion, whether femoral closure devices were deployed and distal pulse examination.

TEVAR-associated perioperative mortality is variable, depending on the acuity and indication of presentation and the patient's comorbidities. As with open surgical repair, TEVAR potentially affects multiple organ systems (Figure 1), hence detailed multidisciplinary attention is necessary for optimal outcomes (6).

\section{Neurological considerations}

\section{Stroke}

The pooled incidence of post-TEVAR stroke reported in a meta-analysis of ten studies and 2,594 patients was $4.1 \%$ (7). In the five studies that characterized LSA coverage, the incidence of stroke was $3.2 \%$ when the LSA was uncovered; when the LSA was covered, the incidence of stroke was lower when the LSA was revascularized (5.3\%) versus not revascularized $(8.0 \%)$. Conversely, another analysis of 2,346 patients found that the stroke incidence was higher (7.5\%) with concomitant revascularization of the LSA than without (4.4\%) and notably higher mortality (24\%) after the stroke; risk factors included mobile atheroma in the aorta and previous stroke (8). A study of stroke after TEVAR (9) found a perioperative stroke rate of $3.8 \%$, with similar distributions in the anterior and posterior cerebral circulation; all strokes were embolic in origin. Patients with chronic kidney disease and previous strokes were at highest risk.

Clinically, if a patient awakes from anesthesia with a focal neurological deficit, immediate brain imaging and neurological consultation is initiated to confirm that no hemorrhagic event necessitating neurosurgical intervention has occurred. Embolic strokes are managed supportively.

\section{Spinal cord deficit (SCD)}

SCD may be the most devastating TEVAR-related complication. A large meta-analysis reported that SCD incidence after TEVAR was $4 \%$ for endovascular repair, including $2 \%$ for DTAAs and $9 \%$ for TAAAs, with no difference between open and endovascular approaches when stratified by aneurysm extension (10).

TEVAR-associated SCD risk factors include extent of aortic coverage, LSA coverage, lengthy procedures with large-bore iliofemoral sheaths, perioperative hypotension, previous infrarenal aortic aneurysm repair, female sex and renal insufficiency (11). Risk for SCD may be reduced by using CSF drainage catheters, pharmacological adjuncts and hemodynamic management (Table 1). 


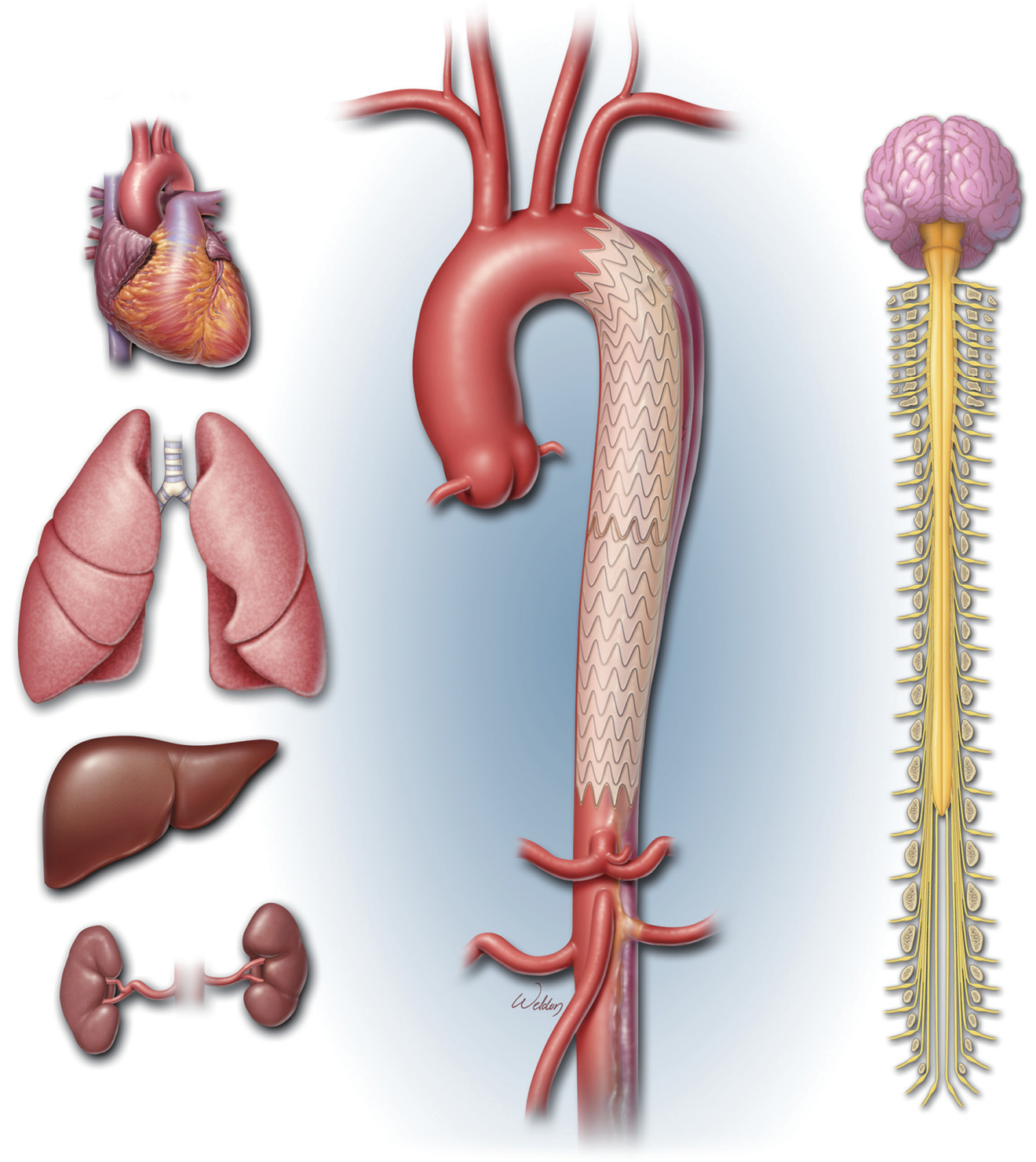

Figure 1 Major organ systems potentially affected after thoracoabdominal aortic aneurysm repair: cardiac, pulmonary, hepatic, renal and neurological (from left to right) (6). Printed with permission from Baylor College of Medicine.

\section{Cerebrospinal fluid drainage}

Although the benefits of CSF drainage in TEVAR procedures are not as clear for isolated DTAAs as they are for more extensive repairs, current guidelines do recommend CSF drainage for patients at risk of SCD during TEVAR $(12,13)$. In our practice, this includes patients with planned full coverage of the descending thoracic aorta, those with previous abdominal aortic replacement (either endovascular or open) and those of specific concern.
Drainage is facilitated by our anesthesiology colleagues, who have gained extensive experience during open TAAA procedures. The anesthesiologist routinely inserts a 14-gauge needle into the intrathecal space below the L2-L3 level and advances it $8-10 \mathrm{~cm}$ within the space. The drain is not placed until the end of the repair in hemodynamically unstable patients or in urgent cases when there is no time for preoperative CSF drainage (these patients may be awakened and assessed for leg strength post-TEVAR). CSF 


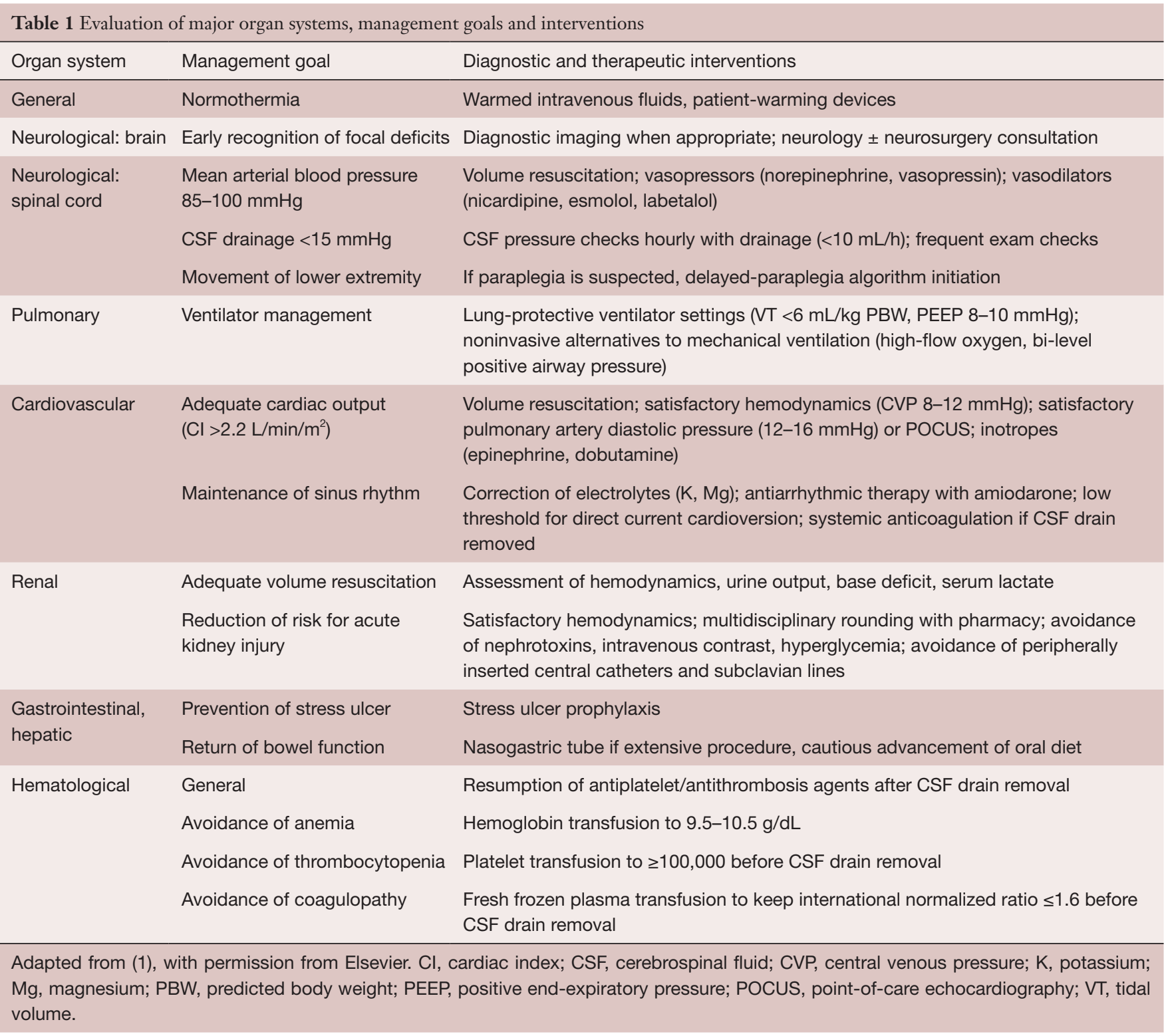

drains are only placed in patients of concern for SCD.

The aim of CSF drainage is to achieve satisfactory spinal cord perfusion pressure [the difference between the mean arterial pressure (MAP) and CSF pressure]. We target a MAP of 85-100 $\mathrm{mmHg}$ and a CSF pressure of $10-15 \mathrm{mmHg}$ to achieve a spinal cord perfusion pressure $\geq 70 \mathrm{mmHg}$. We avoid draining more than $10 \mathrm{~mL}$ per hour, $25 \mathrm{~mL}$ per four hours or $150 \mathrm{~mL}$ per 24 hours, as catastrophic central nervous system bleeding events can result from excessive drainage. Head elevation beyond $30^{\circ}$ during mechanical ventilation is avoided as excessive elevation can cause CSF and cerebral perfusion pressure fluctuations.

Typically, the CSF drain is removed 24-48 hours after TEVAR, depending on repair complexity and whether reliable neurological examination is possible. We clamp the drain for six hours and conduct frequent neurological exams before removal. Proximal leg strength assessment should require the patient to raise their knee off the bed; foot dorsiflexion or hip rotation is insufficient. After drain removal, the patient remains on bedrest for four hours, with periodic neurological checks followed by assisted transfer to a chair. 
Drainage complications are categorized as severe (intracranial hemorrhage, subarachnoid hemorrhage, epidural hematoma, meningitis, catheter or drainagerelated neurological deficit), moderate (spinal headache, CSF leak requiring intervention, e.g., blood patch, drain fracture not requiring surgery), or mild (bloody spinal fluid, puncture-site bleeding, drain fracture, CSF leak not requiring intervention, catheter dislodgment or occlusion). A large meta-analysis (10) reported complication rates after endovascular DTAA or TAAA repair as severe $(4.6 \%)$, moderate $(0.7 \%)$ and mild $(3.2 \%)$. We found that approximately $10 \%$ of patients experienced headaches with CSF drainage after open TAAA repair. Headache is initially managed with caffeine ingestion, and approximately onethird of these patients require an epidural blood patch for symptom resolution (14).

If severe headache is accompanied by nausea and vomiting, CT imaging should be obtained expeditiously to exclude intracerebral hemorrhage. Asymmetric weakness may require imaging to assess for spinal hematoma. Urgent neurosurgical intervention is advised if a hematoma is present.

\section{Delayed paraplegia or paraparesis}

Patients can awaken from anesthesia with full motor function but later develop paraplegia or paraparesis, which is associated with greater risk for permanent disability and death. Delayed paraplegia is hypothesized by some to follow a 'second hit', whereby the 'first hit' occurs during surgery, leaving the spinal cord vulnerable, and the 'second hit' is caused by a hypoperfusion-induced ischemic event during the perioperative period (15). Much of our knowledge about delayed paraplegia is based on open repairs, with one study showing that in patients who developed delayed paraplegia after open TAAA repair, the mean MAP was $74 \mathrm{mmHg}$ at diagnosis, but $95 \mathrm{mmHg}$ during recovery (16).

Rescue maneuvers to increase spinal cord perfusion pressure and maximize oxygen delivery should be promptly initiated if delayed paraplegia or paraparesis develops (Figure 2) (6). The initial goal is to ensure a MAP of 90-100 mmHg. If a CSF drain is present, CSF pressure is reduced to $10-12 \mathrm{mmHg}$ and drainage is increased up to $15 \mathrm{~mL}$ per hour or $50 \mathrm{~mL}$ per four hours. If no CSF drain is present, an anesthesiologist will quickly insert a new drainage catheter. Next, the patient is placed in the Trendelenburg position and the target MAP is increased to $100-115 \mathrm{mmHg}$. Adequate volume resuscitation targets a central venous pressure of $10-12 \mathrm{mmHg}$ and a hemoglobin level of 9.5-10.5 g/dL. Excessive central venous pressure could raise intracerebral and CSF pressures and is thus avoided. Diligent physician monitoring is critical during rescue; increasing MAP and lowering CSF pressure must be done gradually to increase the likelihood of neurological recovery.

Adjunctive agents (e.g., intravenous mannitol $12.5 \mathrm{~g}$, dexamethasone $10 \mathrm{mg}$ ) can be given every 12 hours for 48 hours. If no improvement is evident after 6-12 hours, rescue doses of intravenous lidocaine $100 \mathrm{mg}$ and magnesium sulfate $4 \mathrm{~g}$ are administered. Glucocorticoid administration may induce hyperglycemia, thus close glucose monitoring is required and insulin infusion may be necessary. Similar management algorithms used in other institutions differ slightly from ours but agree on the broad principles $(17,18)$.

Patients who awaken with paraplegia or paraparesis are unlikely to recover. For these patients, we increase the MAP to $100-110 \mathrm{mmHg}$, drain the CSF to approximately $10-15 \mathrm{mmHg}$, and treat with mannitol and dexamethasone. Because paraplegia lasting longer than 48 hours is probably permanent, venous thromboembolism prevention measures and bowel and bladder management protocols should be initiated.

\section{Hemodynamic management}

Blood pressure should be maintained between a 'floor' (lowest acceptable blood pressure before SCD risk increases) and a 'ceiling' (highest acceptable blood pressure before no further SCD prevention benefit remains and the patient risks harmful hypertension). Indications for TEVAR will influence the blood pressure target. For degenerative aneurysms, hypotension is the primary concern, whereas relative hypertension is well tolerated. Conversely, for TBAD with residual dissection, hypertension-induced exacerbation of the uncovered residual dissection is a risk. Stabilizing hemodynamics, avoiding labile blood pressure and hypotension, and maintaining satisfactory MAP are critical measures for avoiding SCD.

Patients are occasionally hypothermic $\left(33-34{ }^{\circ} \mathrm{C}\right)$ when they arrive in the intensive care unit (ICU). If the patient is shivering, demand ischemia and lower cardiac output can risk SCD. Rewarming to normothermia induces vasodilation and decreases MAP, which should be treated. Our typical vasopressor strategy is norepinephrine followed by vasopressin. We usually aim to maintain hemoglobin above $9 \mathrm{~g} / \mathrm{dL}$.

Moderate hypertension (systolic blood pressure of 140 


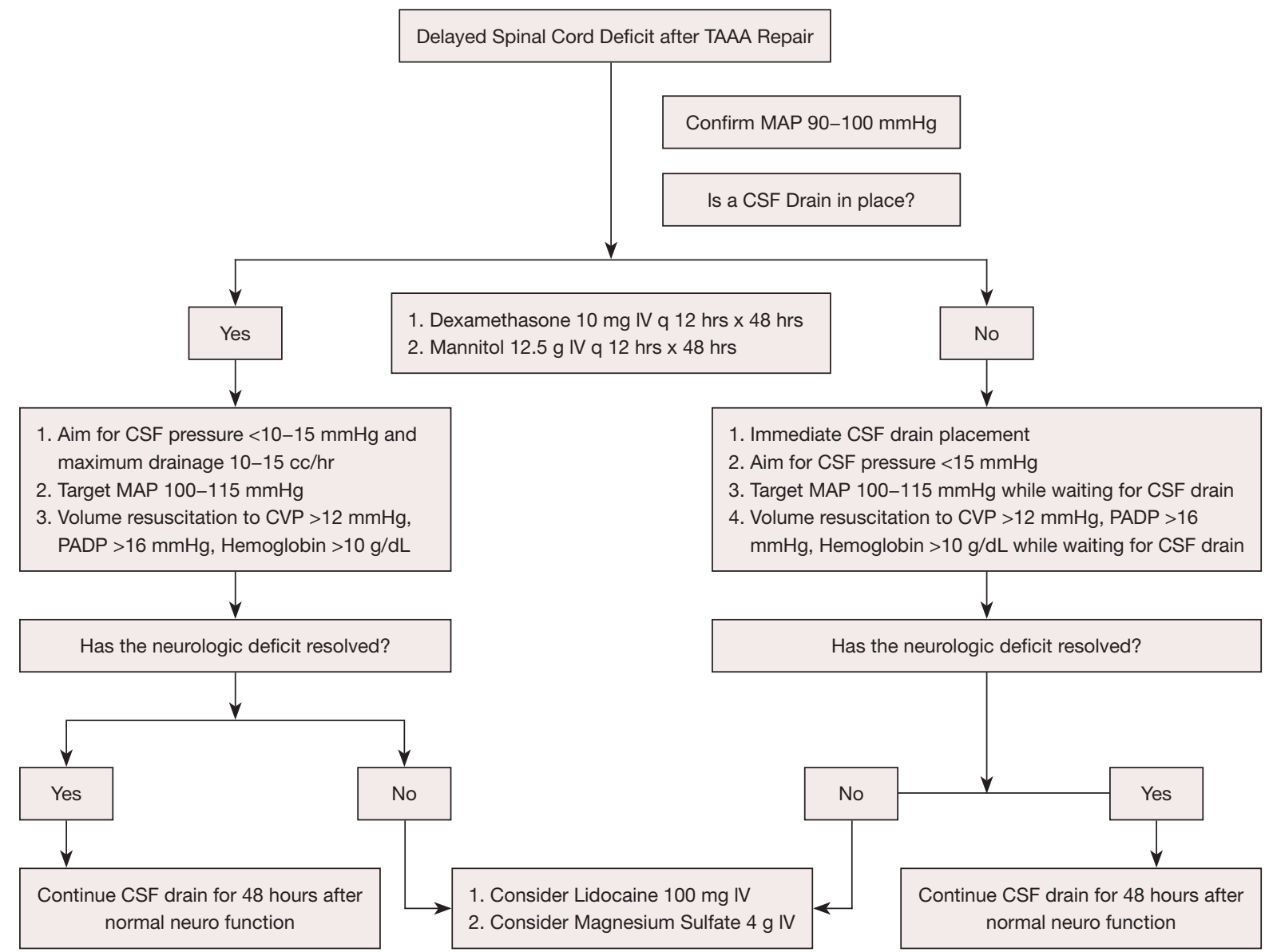

Figure 2 Algorithm for managing delayed paraplegia after thoracoabdominal aortic aneurysm repair (6); also used for managing delayed paraplegia after TEVAR. Reprinted from (6), with permission from Elsevier. CSF, cerebrospinal fluid; CVP, central venous pressure; IV, intravenous; MAP, mean arterial pressure; PADP, pulmonary artery diastolic pressure; TAAA, thoracoabdominal aortic aneurysm; TEVAR; thoracic endovascular aortic repair.

$150 \mathrm{mmHg}$ ) is not treated aggressively. We recommend waiting 4-6 weeks after TEVAR before strict hypertension control. Severe hypertension (MAP $>120 \mathrm{mmHg}$ ) can cause complications, especially for patients with TBAD. We use systemic vasodilators (labetalol followed by nicardipine or esmolol) to treat severe hypertension.

\section{Respiratory considerations}

Patients who undergo elective TEVAR are typically extubated in the operating room, whereas in urgent cases, patients may require ongoing mechanical ventilation. We target lung-protective ventilation with a goal tidal volume of $6 \mathrm{~mL} / \mathrm{kg}$ of predicted body weight and a positive end-expiratory pressure of $8-10 \mathrm{mmHg}$. In borderline candidates, high-flow oxygen and bi-level positive airway pressure can facilitate extubation. After extubation, coughing, deep breathing, bronchodilator therapy and other pulmonary toilet measures are encouraged, especially in patients with pre-existing moderate to severe pulmonary comorbidities (Table 1). In a Nationwide Inpatient Sample study with 8,255 descending thoracic aortic aneurysm repair patients and 712 TEVAR patients, unadjusted postoperative respiratory complication rates were $5.6 \%$ vs. $2.4 \%$, respectively (19).

\section{Cardiovascular considerations}

An arterial line and central venous catheter are typically used for hemodynamic monitoring. For more extensive 
repairs, a pulmonary artery catheter and continuous cardiac output monitor may be used if the preoperative left ventricular ejection fraction is $\leq 35 \%$. Adequate cardiac output is achieved with volume resuscitation and, if needed, epinephrine for augmenting both cardiac output and blood pressure. During resuscitation, we generally monitor cardiac output (if available), mixed venous oxygen saturation, serum lactate and urine output.

Close monitoring for postoperative atrial fibrillation is necessary, although the reported incidence is considerably lower after TEVAR (9\%) (20) than after open TAAA repair or routine cardiac surgery (approximately $23 \%$ for both) (21). Postoperative atrial fibrillation may cause hypotension secondary to loss of atrial kick, which in turn can cause SCD. Preventive strategies include electrolyte monitoring and correction. Rate control with atrioventricular nodal agents can risk hypotension and SCD, so a cautious approach is advised; we prefer amiodarone for aggressive rhythm control. For TEVAR patients, who often have preexisting respiratory disease, amiodarone during the index hospitalization is well tolerated and unlikely to produce pulmonary toxicity (22). Cardioversion may be appropriate for patients experiencing prolonged or symptomatic dysrhythmias. Sustained postoperative atrial fibrillation may warrant anticoagulation, however this decision is individualized, with recent preference leaning towards use of direct oral anticoagulants.

Post-TEVAR vascular examinations should be conducted in the operating room and postoperatively. Major vascular complications can occur in up to $12 \%$ of patients with iliofemoral vascular size discrepancies (23). These complications have been associated with a $13 \%$ mortality rate and require prompt recognition for prevention and optimal management. Early intervention may be necessary for limb salvage to avoid compartment syndrome. Ischemic complications can be caused by arterial thrombosis, embolism, aortic dissection or endograft malpositioning (24). Device-related complications (endoleaks, endograft migration or collapse, kinking or stenosis of an endograft limb, graft infection) require vigilant monitoring, as $19-24 \%$ of cases require some type of secondary intervention (24). Finally, retrograde type A aortic dissection after TEVAR occurs in 1-3\% of cases and is associated with a mortality rate of almost $40 \%(25,26)$.

Postimplantation syndrome is a unique complication of endovascular repair that occurs in $13-60 \%$ of patients (24). Thought to be an inflammatory, immune-mediated response, it is characterized by fever, leukocytosis and elevated levels of inflammatory markers such as C-reactive protein. Pleural effusions have been noted in $37-73 \%$ of TEVAR cases (27). Treatment is largely supportive (aspirin and surveillance); antibiotics have not been proven beneficial.

\section{Renal considerations}

Preventing AKI is a major priority after TEVAR. Endovascular TAAA repair was associated with a high rate $(32 \%)$ of postoperative renal insufficiency, defined as a $50 \%$ increase in serum creatinine (28) consistent with moderate AKI, which compromises survival (29).

The incidence of AKI in open TAAA repair series ranges from $5-29 \%$ (depending on how AKI is defined), with 4-17\% needing renal replacement therapy (RRT) (29). In TEVAR, the published incidence of AKI is approximately $10 \%$, with less need for RRT (30). In severe AKI, RRT is initiated for volume overload, refractory acidemia or electrolyte abnormalities (31). Fundamental AKI management guidelines include routine preventive strategies (e.g., multidisciplinary rounding with pharmacists) (32).

The risk of contrast nephropathy after TEVAR is related to higher contrast volume and aortic dissection extending into the renal arteries (33). To reduce this risk, intravenous hydration is administered before imaging commences (34). Due to the risk of hyperchloremic metabolic acidosis, normal saline is avoided in favor of isotonic solutions for volume resuscitation.

Meticulous care of vascular access catheters is necessary to reduce the risk of catheter-associated bloodstream infections that could infect the TEVAR graft. For this reason, peripherally inserted central catheter lines are avoided in patients with AKI (35). When longer-term RRT appears necessary, conversion to a tunneled dialysis catheter can reduce the infection risk associated with temporary catheters. Future dialysis access needs should be considered in patients with chronic kidney disease (Table 1).

\section{Gastrointestinal and nutritional considerations}

Most patients are able to tolerate a regular diet within 24 hours. Patients who undergo TEVAR for TAAA or TBAD occasionally benefit from continued nasogastric tube drainage after extubation. With TBAD, careful vigilance is needed to monitor for gut malperfusion (36), as indicators (e.g., acidemia, increased intravenous fluid requirements) may be subtle. In patients who continue to be mechanically 
ventilated, enteral feeds are begun 48-72 hours after surgery. Routine stress ulcer prophylaxis should be administered, and gastrointestinal bleeding not responding to higher-dose proton-pump inhibitors or lavage should be evaluated endoscopically.

\section{Bleeding and hematological considerations}

Early postoperative bleeding from femoral access sites can induce hemorrhagic shock. An unexplained drop in hemoglobin may necessitate CT imaging to identify hemorrhage in the chest or abdomen, especially if hypotension persists. Vigilance is necessary, and early return to the operating room for exploration, evacuation and surgical control may be needed.

For patients who were taking warfarin for previous mechanical valve prostheses, we typically resume warfarin 2-3 days postoperatively without bridging anticoagulation, given the potential risk for bleeding from the CSF drainage site. For patients who may need additional procedures (e.g., tracheostomy) due to a prolonged ICU course, unfractionated heparin is started 48-72 hours after the CSF drain is removed. Oral anticoagulation is resumed when no further surgical procedures will be needed. Chemical prophylaxis against venous thromboembolism is initiated 24 hours after CSF drain removal. Aspirin is started the day of CSF drain removal, whereas $\mathrm{P}_{2} \mathrm{Y}_{12}$ receptor blockers are resumed 24 hours after drain removal.

\section{Miscellaneous considerations}

Early mobility and removal of intravascular or urinary catheters is encouraged. Postoperative aortic imaging provides a baseline for ongoing surveillance but is best done after serum creatinine level normalizes. Nonetheless, more than half of TEVAR patients are lost to follow-up and not compliant with routine surveillance imaging (37). Continued communication with primary and referring cardiologists is necessary to ensure optimal long-term survival.

\section{Conclusions}

The prevention, early detection and appropriate management of post-TEVAR complications depends on diligent, multidisciplinary care. Close attention across the perioperative, intraoperative and postoperative treatment phases makes achieving successful outcomes more likely.

\section{Acknowledgments}

We appreciate the editorial support of Jeanie Woodruff, BS, ELS, from the Texas Heart Institute's Scientific Publications Department, and the graphic artwork of Scott Weldon, MA, CMI, FAMI, of the Division of Cardiothoracic Surgery at Baylor College of Medicine.

Funding: None.

\section{Footnote}

Conflicts of Interest: Dr. SC has participated in advisory boards for Edwards Lifesciences \& La Jolla Pharmaceutical Corp. Dr. OP provides consultation for and participates in clinical trials with Medtronic and W.L. Gore \& Associates. Dr. JSC participates in clinical trials with and/or consults for Terumo Aortic, Medtronic, and W.L. Gore \& Associates and receives royalties and grant support from Terumo Aortic. The other author has no conflicts of interest to declare.

Open Access Statement: This is an Open Access article distributed in accordance with the Creative Commons Attribution-NonCommercial-NoDerivs 4.0 International License (CC BY-NC-ND 4.0), which permits the noncommercial replication and distribution of the article with the strict proviso that no changes or edits are made and the original work is properly cited (including links to both the formal publication through the relevant DOI and the license). See: https://creativecommons.org/licenses/by-nc-nd/4.0/.

\section{References}

1. Chatterjee S, Preventza O, Orozco-Sevilla V, et al. Critical care management after open thoracoabdominal aortic aneurysm repair. J Cardiovasc Surg (Torino) 2021;62:220-9.

2. Fleisher LA, Fleischmann KE, Auerbach AD, et al. 2014 ACC/AHA guideline on perioperative cardiovascular evaluation and management of patients undergoing noncardiac surgery: a report of the American College of Cardiology/American Heart Association Task Force on practice guidelines. J Am Coll Cardiol 2014;64:e77-137.

3. Chatterjee S, Casar JG, LeMaire SA, et al. Perioperative care after thoracoabdominal aortic aneurysm repair: The Baylor College of Medicine experience. Part 1: Preoperative considerations. J Thorac Cardiovasc Surg 2021;161:693-8. 
4. Tan CW, Wall M, Rosengart TK, et al. How to bridge? Management of anticoagulation in patients with mechanical heart valves undergoing noncardiac surgical procedures. J Thorac Cardiovasc Surg 2019;158:200-3.

5. Chatterjee S, Shake JG, Arora RC, et al. Handoffs From the Operating Room to the Intensive Care Unit After Cardiothoracic Surgery: From The Society of Thoracic Surgeons Workforce on Critical Care. Ann Thorac Surg 2019;107:619-30.

6. Chatterjee S, Casar JG, LeMaire SA, et al. Perioperative care after thoracoabdominal aortic aneurysm repair: The Baylor College of Medicine experience. Part 2: Postoperative management. J Thorac Cardiovasc Surg 2021;161:699-705.

7. von Allmen RS, Gahl B, Powell JT. Editor's Choice Incidence of Stroke Following Thoracic Endovascular Aortic Repair for Descending Aortic Aneurysm: A Systematic Review of the Literature with Meta-analysis. Eur J Vasc Endovasc Surg 2017;53:176-84.

8. Varkevisser RRB, Swerdlow NJ, de Guerre LEVM, et al. Thoracic Endovascular Aortic Repair With Left Subclavian Artery Coverage Is Associated With a High 30-Day Stroke Incidence With or Without Concomitant Revascularization. J Endovasc Ther 2020;27:769-76.

9. Ullery BW, McGarvey M, Cheung AT, et al. Vascular distribution of stroke and its relationship to perioperative mortality and neurologic outcome after thoracic endovascular aortic repair. J Vasc Surg 2012;56:1510-7.

10. Gaudino M, Khan FM, Rahouma M, et al. Spinal cord injury after open and endovascular repair of descending thoracic and thoracoabdominal aortic aneurysms: A metaanalysis. J Thorac Cardiovasc Surg 2020. [Epub ahead of print]. doi: 10.1016/j.jtcvs.2020.04.126.

11. Preventza O, Wheatley GH 3rd, Williams J, et al. Identifying paraplegia risk associated with thoracic endografting. Asian Cardiovasc Thorac Ann 2009;17:568-72.

12. Czerny M, Pacini D, Aboyans V, et al. Current options and recommendations for the use of thoracic endovascular aortic repair in acute and chronic thoracic aortic disease: an expert consensus document of the European Society for Cardiology (ESC) Working Group of Cardiovascular Surgery, the ESC Working Group on Aorta and Peripheral Vascular Diseases, the European Association of Percutaneous Cardiovascular Interventions (EAPCI) of the ESC and the European Association for Cardio-Thoracic Surgery (EACTS). Eur J Cardiothorac Surg 2021;59:65-73.

13. Hiratzka LF, Bakris GL, Beckman JA, et al. 2010

\section{ACCF/AHA/AATS/ACR/ASA/SCA/SCAI/SIR/STS/}

SVM Guidelines for the diagnosis and management of patients with thoracic aortic disease. A Report of the American College of Cardiology Foundation/American Heart Association Task Force on Practice Guidelines, American Association for Thoracic Surgery, American College of Radiology, American Stroke Association, Society of Cardiovascular Anesthesiologists, Society for Cardiovascular Angiography and Interventions, Society of Interventional Radiology, Society of Thoracic Surgeons, and Society for Vascular Medicine. J Am Coll Cardiol 2010;55:e27-e129.

14. Youngblood SC, Tolpin DA, LeMaire SA, et al. Complications of cerebrospinal fluid drainage after thoracic aortic surgery: a review of 504 patients over 5 years. J Thorac Cardiovasc Surg 2013;146:166-71.

15. Tanaka A, Safi HJ, Estrera AL. Current strategies of spinal cord protection during thoracoabdominal aortic surgery. Gen Thorac Cardiovasc Surg 2018;66:307-14.

16. Maniar HS, Sundt TM 3rd, Prasad SM, et al. Delayed paraplegia after thoracic and thoracoabdominal aneurysm repair: a continuing risk. Ann Thorac Surg 2003;75:113-9; discussions 119-20.

17. Estrera AL, Sheinbaum R, Miller CC, et al. Cerebrospinal fluid drainage during thoracic aortic repair: safety and current management. Ann Thorac Surg 2009;88:9-15; discussion 15 .

18. Maurel B, Delclaux N, Sobocinski J, et al. The impact of early pelvic and lower limb reperfusion and attentive peri-operative management on the incidence of spinal cord ischemia during thoracoabdominal aortic aneurysm endovascular repair. Eur J Vasc Endovasc Surg 2015;49:248-54.

19. Hughes K, Guerrier J, Obirieze A, et al. Open versus endovascular repair of thoracic aortic aneurysms: a Nationwide Inpatient Sample study. Vasc Endovascular Surg 2014;48:383-7.

20. Bub GL, Greenberg RK, Mastracci TM, et al. Perioperative cardiac events in endovascular repair of complex aortic aneurysms and association with preoperative studies. J Vasc Surg 2011;53:21-27.e1-2.

21. Dolapoglu A, Volguina IV, Price MD, et al. Cardiac Arrhythmia After Open Thoracoabdominal Aortic Aneurysm Repair. Ann Thorac Surg 2017;104:854-60.

22. Wolkove N, Baltzan M. Amiodarone pulmonary toxicity. Can Respir J 2009; 16:43-8.

23. Vandy FC, Girotti M, Williams DM, et al. Iliofemoral complications associated with thoracic endovascular aortic 
repair: frequency, risk factors, and early and late outcomes. J Thorac Cardiovasc Surg 2014;147:960-5.

24. Daye D, Walker TG. Complications of endovascular aneurysm repair of the thoracic and abdominal aorta: evaluation and management. Cardiovasc Diagn Ther 2018;8:S138-56.

25. Chen Y, Zhang S, Liu L, et al. Retrograde Type A Aortic Dissection After Thoracic Endovascular Aortic Repair: A Systematic Review and Meta-Analysis. J Am Heart Assoc 2017;6:004649.

26. Preventza O, Garcia A, Moeller K, et al. Retrograde Ascending Aortic Dissection After Thoracic Endovascular Aortic Repair for Distal Aortic Dissection or With Zone 0 Landing: Association, Risk Factors, and True Incidence. Ann Thorac Surg 2015;100:509-15.

27. Eggebrecht H, Mehta RH, Metozounve H, et al. Clinical implications of systemic inflammatory response syndrome following thoracic aortic stent-graft placement. J Endovasc Ther 2008; 15:135-43.

28. Chang CK, Chuter TA, Niemann CU, et al. Systemic inflammation, coagulopathy, and acute renal insufficiency following endovascular thoracoabdominal aortic aneurysm repair. J Vasc Surg 2009;49:1140-6.

29. Chatterjee S, LeMaire SA, Amarasekara HS, et al. Early-Stage Acute Kidney Injury Adversely Affects Thoracoabdominal Aortic Aneurysm Repair Outcomes. Ann Thorac Surg 2019;107:1720-6.

30. Pisimisis GT, Khoynezhad A, Bashir K, et al. Incidence

Cite this article as: Chatterjee S, Preventza O, OrozcoSevilla V, Coselli JS. Perioperative management of patients undergoing thoracic endovascular repair. Ann Cardiothorac Surg 2021;10(6):768-777. doi: 10.21037/acs-2021-taes-74 and risk factors of renal dysfunction after thoracic endovascular aortic repair. J Thorac Cardiovasc Surg 2010;140:S161-7.

31. Küllmar M, Zarbock A, Engelman DT, et al. Prevention of Acute Kidney Injury. Crit Care Clin 2020;36:691-704.

32. Khwaja A. KDIGO clinical practice guidelines for acute kidney injury. Nephron Clin Pract 2012;120:c179-84.

33. Li X, Zhang W, Liu J, et al. Contrast-Induced Kidney Nephropathy in Thoracic Endovascular Aortic Repair: A 2-Year Retrospective Study in 470 Patients. Angiology 2020;71:242-8.

34. Kwok CS, Pang CL, Yeong JK, et al. Measures used to treat contrast-induced nephropathy: overview of reviews. Br J Radiol 2013;86:20120272.

35. Hoggard J, Saad T, Schon D, et al. Guidelines for venous access in patients with chronic kidney disease. A Position Statement from the American Society of Diagnostic and Interventional Nephrology, Clinical Practice Committee and the Association for Vascular Access. Semin Dial 2008;21:186-91.

36. Yamamoto M, Tashiro M, Noguchi T, et al. Visceral malperfusion after thoracic endovascular aortic repair for type $\mathrm{B}$ aortic dissection in a post-abdominal aortic grafting patient. J Cardiol Cases 2018;18:92-4.

37. Kret MR, Azarbal AF, Mitchell EL, et al. Compliance with long-term surveillance recommendations following endovascular aneurysm repair or type B aortic dissection. J Vasc Surg 2013;58:25-31. 